\title{
Atom Indonesia
}

\section{Simulations of Beam Quality in a $13 \mathrm{MeV}$ PET Cyclotron}

\author{
A. Pramudita*, E. Mulyani and I.A. Kudus \\ Center for Accelerator Science and Technology, National Nuclear Energy Agency, \\ Jl. Babarsari, Yogyakarta 55281, Indonesia
}

\section{ARTICLE INFO}

\section{Article history:}

Received 12 March 2014

Received in revised form 30 June 2015

Accepted 01 July 2015

\section{Keywords:}

$13 \mathrm{MeV}$ cyclotron

Fourth harmonics

Negative hydrogen ion $\left(\mathrm{H}^{-}\right)$

Runge-Kutta (RK4)

Scilab 5.3.3

Opera-3d/TOSCA

\begin{abstract}
A B S T R A C T
Simulation of the trajectories of negative hydrogen ion $\left(\mathrm{H}^{-}\right)$beam in a $13 \mathrm{MeV}$ PET cyclotron (DECY-13) were carried out by using the Runge-Kutta (RK4) approximation method and Scilab 5.4.1. The magnetic and electric fields were calculated using Opera-3d/TOSCA softwares at $1 \mathrm{~mm}$ resolution. The cyclotron is of a fourth-harmonics type, meaning that the acceleration occurs four times per cycle, with a radiofrequency (RF) field of $77.66 \mathrm{MHz}$ frequency and $40 \mathrm{kV}$ amplitude. The calculations and simulations show that the maximum distance between the ion source and the puller is about $6 \mathrm{~mm}$, while the maximum width of the beam at $13 \mathrm{MeV}$ is about $10 \mathrm{~mm}$, and the initial phase between the RF field and the beam ranges from $-10^{\circ}$ to $10^{\circ}$, with a yield of about $10 \%$ of the beam from the ion source getting accelerated to $13 \mathrm{MeV}$.
\end{abstract}

(C) 2015 Atom Indonesia. All rights reserved

\section{INTRODUCTION}

The activities at the Center for Accelerator Science and Technology, National Nuclear Energy Agency (BATAN), include developmental activities of accelerator technology, and one of those activities is the development of a $13 \mathrm{MeV}$ proton cyclotron for PET (Positron Emission Tomography). This activity is named DECY-13 (Development of Experimental Cyclotron in Yogyakarta - $13 \mathrm{MeV}$ ), and is planned to be completed in 2019. To design a cyclotron, it is important to understand the proton beam's properties, including its trajectory from the ion source to the target. The proton beam's trajectory is determined by the distribution of the RF (radiofrequency) electric field which accelerates the beam, the distribution of the magnetic field which bends and focuses the beam, and the initial position and phase of the beam. The distributions of the electric and magnetic fields in three dimensions can be calculated by using the Opera-3d software and the TOSCA module (for static electric and magnetic fields[2]), the Soprano module (for RF electric

\footnotetext{
* Corresponding author.

E-mail address: pramudita@batan.go.id

DOI: http://dx.doi.org/10.17146/aij.2015.411
}

fields), and the RELAX3D software (for static electric fields).

The proton beam trajectory simulations had been carried out by using Scilab, an open source software similar to the commercial software Matlab. The simulations can be used to evaluate the design of the cyclotron to determine whether it properly functions and reaches the required beam energy and quality, or still requires further design improvements.

The simulations of the beam's trajectories in the cyclotron were carried out in 2012 using Scilab 5.3.3 for $\mathrm{H}^{-}$beam trajectories for energies of up to $13 \mathrm{MeV}$. The trajectory calculation was done using the equation of motion $F=d p / d t=m \gamma(d v / d t)$, where $F$ is the Lorentz force, $p$ is the momentum, $m$ is the rest mass, $\gamma$ is the relativistic correction, and $t$ is time, while velocity is represented by $v=d s / d t=v_{\mathrm{o}}$ $+d v$, and position by $x=x_{\mathrm{o}}+v d t$, where $v_{\mathrm{o}}$ and $x_{\mathrm{o}}$ are initial velocity and initial position, respectively. The spatial step $d s$ was fixed at $1 \mathrm{~mm}$, and the same with the precision of the electric and magnetic field simulations. Using this method, it was found the calculated beam energy increase was not linear.

Beam trajectory simulations were also performed in Korea for a cyclotron design by using 
the pwheel program, written in Fortran, which employed a Runge-Kutta numerical algorithm (the RK4) with a fixed time step $d t$. This method has been applied in the program for beam trajectory simulation in Scilab 5.4.1.

Beam dynamics studies in cyclotrons were also conducted in Korea for a $9 \mathrm{MeV}$ [3] and $13 \mathrm{MeV}$ [4] PET cyclotrons, in China for a $10 \mathrm{MeV}$ [5], a $14 \mathrm{MeV}$ [6] and a 75-100 MeV [7] ones, and in Russia for a dedicated $2.5 \mathrm{MeV} / A$ ( $A$ is atomic mass number) heavy ion cyclotron [8]. Except in references 1 and 6 , the general results were presented without much detail as provided in this paper.

\section{THEORY}

The calculation of the trajectory an ion of electric charge $q$ and velocity $v$ is based on the Lorentz force $F$ acting on the charged particle in an electric field $E$ and a magnetic field $B$. The electric field $E$ is a function of position $(x, y, z)$ and time $(t)$, while the magnetic field $B$ is a function of position only. The values of both fields are obtained from calculations, simulations, or measurements (map-ping).

The $x, y$, and $z$ components of the equations of motion can be written as

$$
\begin{aligned}
& d p_{x} / d t=F_{x}=q\left(E_{x}+v_{y} B_{z}-v_{z} B_{y}\right), \\
& d p_{y} / d t=F_{y}=q\left(E_{y}+v_{z} B_{x}-v_{x} B_{z}\right), \\
& d p_{z} / d t=F_{z}=q\left(E_{z}+v_{x} B_{y}-v_{y} B_{x}\right) .
\end{aligned}
$$

The solutions of each component of equation (1), obtained using a fourth-order Runge-Kutta method (RK4), are [9] (with $i=x, y, z$ )

$$
p_{i}\left(t_{\mathrm{o}}+d t\right)=p_{i \mathrm{o}}+\left(k_{i 1}+2 k_{i 2}+2 k_{i 3}+k_{i 4}\right) / 6,
$$

where

$$
\begin{aligned}
k_{i 1} & =F_{i}\left(t_{\mathrm{o}}, p_{\mathrm{o}}\right) d t, \\
k_{i 2} & =F_{i}\left(t_{\mathrm{o}}+d t / 2, p_{\mathrm{o}}+k_{i 1} / 2\right) d t, \\
k_{i 3} & =F_{i}\left(t_{\mathrm{o}}+d t / 2, p_{\mathrm{o}}+k_{i 2} / 2\right) d t, \\
k_{i 4} & =F_{i}\left(t_{\mathrm{o}}+d t, p_{\mathrm{o}}+k_{i 3}\right) d t .
\end{aligned}
$$

After the new momentum $p_{i}$ is obtained, the new velocity $v_{i}=p_{i} / \gamma m$ and the new position $x_{i}=x_{i o}+$ $v_{i} d t$ can be determined.

As the magnetic and electric potential field data are given at $\mathrm{mm}$ accuracy, the field value for each calculated position is rounded to the nearest $\mathrm{mm}$. In trajectory calculation using pwheel, five-point bspline interpolation was employed, and electric potential data was provided at accuracies of $0.25 \mathrm{~mm}, 0.5 \mathrm{~mm}, 1 \mathrm{~mm}, 2 \mathrm{~mm}$, and $4 \mathrm{~mm}$ at a $256 \times 256 \times 5$ data size. In this paper, the bspline interpolation, which is also available in Scilab, was not employed, as it would require much longer calculation times.

\section{CALCULATION}

The program for the $\mathrm{H}^{-}$beam trajectory computation was written in Scilab 5.4.1. The program requires the data of the magnetic field which is loaded using the loadB program. Because of symmetry, it was sufficient to have the data of only $1 / 8$ of the overall field volume of $480 \mathrm{~mm} \times$ $480 \mathrm{~mm} \times 30 \mathrm{~mm}$; the data was obtained from computation using Opera-3d software and TOSCA module[2]. The size of the binary data was about $38 \mathrm{MB}$ for each of the $B_{x}, B_{y}$, and $B_{z}$ components.

The data on the electric potentials at a dee voltage of $40 \mathrm{kV}$ relative to ground was also computed by using the Opera-3d software and the TOSCA module. The data was loaded by using the loadV12 and loadV34 programs. As there is no symmetry, the data must cover the entire field region of $480 \mathrm{~mm} \times 480 \mathrm{~mm} \times 30 \mathrm{~mm}$ dimension. To reduce the size of the data, it was divided into four quadrants, namely V1, V2 (loadV12), V3, and V4 (load V34). The size of the binary data was about $56 \mathrm{MB}$ for each V1, V2, V3, and V4. The electric fields $E_{x}, E_{y}$, and $E_{z}$ for each quadrant were calculated from the potential difference at spatial distance $d s=1 \mathrm{~mm}$ in the direction of each electric field component.

The beam from the ion source (IS) will enter the puller only if it arrives before the puller changes its polarity. Hence it should reach the puller within the duration of less than or equal to $T / 2$, where $T$ is the period of the RF electric field. If the initial velocity of the beam coming out from the IS $\approx 0$, the maximum distance between IS to the puller is approximately $s=1 / 2 a t^{2}=d, a \approx e V / m d$, then $d=(q V / 2 m)^{1 / 2} T / 2$. With $V=40,000$ volt, $T=1 /(77.66 \mathrm{MHz})$, and $m=$ mass of $\mathrm{H}^{-}$, we obtain $d \leq 8.9065 \mathrm{~mm}$. As $V$ varies, the effective value $V /(2)^{1 / 2}$ should be taken, then $d \leq 6.2978 \mathrm{~mm}$ $\approx 6 \mathrm{~mm}$. This $d$ value was used in the simulations cited in this paper.

The beam starts at the center of the ion source slit: $x=17.5 \mathrm{~mm} ; y=9 \mathrm{~mm}$; and $z=0$. The beam initial velocity was approximated by the increase of the beam energy upon travelling $0.1 \mathrm{~mm}$ from the initial posisition. The motion, energy, and position 
were calculated by using equations (1), (2), and (3). The trajectory is plotted in the XY plane, while the energy and vertical position are plotted as the function of turn-number. As the cyclotron operates in the fourth harmonics, each turn-number equals to four cycles of the accelerating radiofrequency electric field. The beam position at each beginning of turn-number is plotted (in black) against the beam trajectory (in green), showing the beam phase at each turn. Fig. 1 shows the beam trajectory and phase with initial phase of $-25^{\circ}$.

Figure 2 shows the energy at each turnnumber. The energy reaches $13.95 \mathrm{MeV}$ at the $100^{\text {th }}$ turn, while the $13 \mathrm{MeV}$ energy is reached at approximately the $92^{\text {nd }}$ turn. Figure 3 shows the vertical position $(z)$ of the beam, which is approximately $12 \mathrm{~mm}$ wide at the $100^{\text {th }}$ turn.

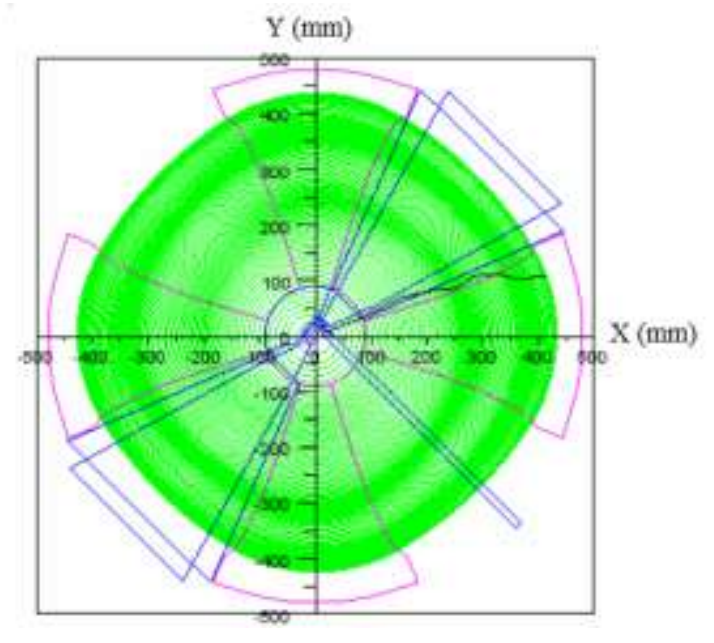

Fig. 1. The beam trajectory (green) for 100 turns with an initial beam phase of $-25^{\circ}$. The black curve at the lower first quadrant shows the beam phase at the beginning of each turn-number.

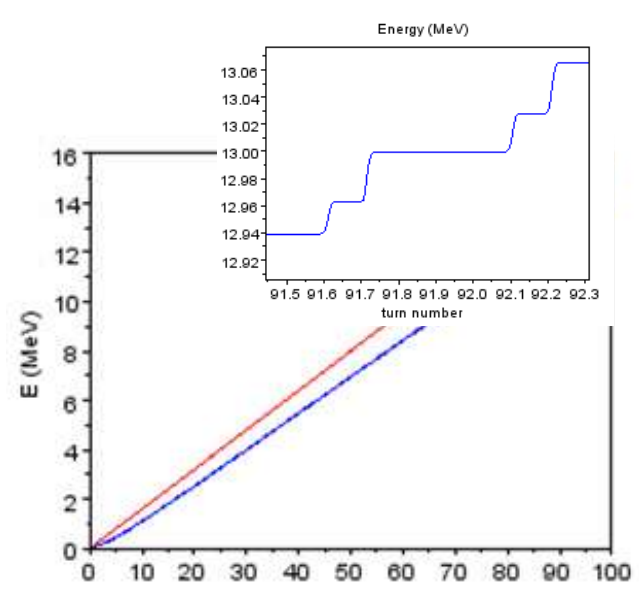

Fig. 2. The beam energy (blue) for 100 turns with an initial beam phase of $-25^{\circ}$. The red curve shows the maximum energy $(4 \times 40 \mathrm{keV})$ reachable at each turn. Enlarged graph (inset) shows that $13 \mathrm{MeV}$ is reached at approximately turn number 92 .

Figure 4 is the plot of energy and $y$ position which shows the position of the beam at $\mathrm{Y}^{+}$axis when the closest beam energy of $13 \mathrm{MeV}$ is reached. The stripper to change the $\mathrm{H}^{-}$beam into an $\mathrm{H}^{+}$beam, hence changing the direction of the beam outward for extraction, is located to the left just after the $\mathrm{Y}^{+}$axis. Since the outermost trajectory is that of the $100^{\text {th }}$ turn, Fig. 4 shows that the beam energy of $13 \mathrm{MeV}$ is reached by the $92^{\text {nd }}$ turn, consistent with Fig. 2. It also shows that $y_{\max } \approx 425 \mathrm{~mm}$ at the $92^{\text {nd }}$ turn or at the beam energy of $13 \mathrm{MeV}$. Different values of $y_{\max }$ for different initial points of the beam will provide estimates on the horizontal width of the beam near the stripper. The beam's vertical width can be estimated from Fig. 3 .

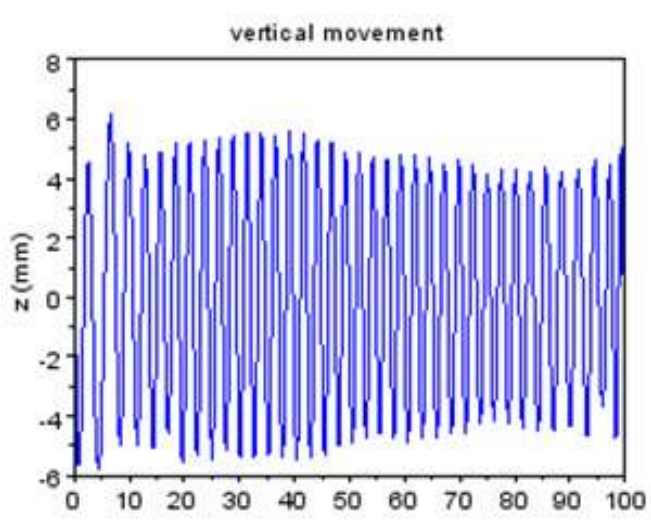

Fig. 3. The beam vertical position (blue) for 100 turns with initial beam phase $-25^{\circ}$. The curve shows that the maximum vertical width of the beam is about $12 \mathrm{~mm}$ at the $100^{\text {th }}$ turn.

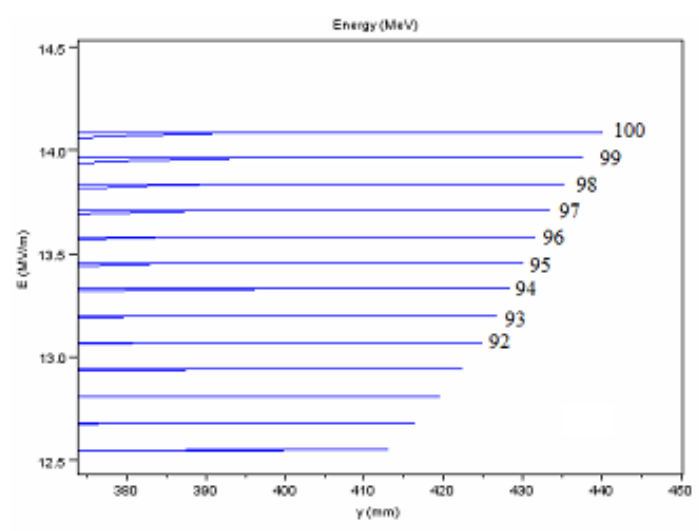

Fig. 4. The beam energy (MeV) is plotted against $y$ position (mm) for 100 turns (shown here for the final 12 turns) with initial beam phase $-25^{\circ}$, showing that $13 \mathrm{MeV}$ is reached at the $92^{\text {nd }}$ turn where $y_{\max } \approx 425 \mathrm{~mm}$. At the 91 st turn the energy is still less than $13 \mathrm{MeV}$.

\section{RESULTS AND DISCUSSION}

After simulations with various initial beam phases, it was determined that the beam reached the intended $13 \mathrm{MeV}$ energy if the initial phases were 
between $-10^{\circ}$ and $10^{\circ}$. The position of the beam trajectory in the puller was also better centered if the slit of the ion source was shifted $1 \mathrm{~mm}$ to the right, as shown in Fig. 5. The slit center's position became $x=18.67 \mathrm{~mm}, y=9.23 \mathrm{~mm}, z=0$.

The slit of the ion source for DECY-13 is having a size of $4 \mathrm{~mm}$ height and $0.55 \mathrm{~mm}$ width. All beam trajectory coming out from the slit of the ion source were represented as starting at 9 points: at $z=-2,0,2 \mathrm{~mm}, 3$ points each along vertical axis of the slit $(x, y=18.67,9.23)$, and 3 points each $0.275 \mathrm{~mm}$ to the left $(x, y=18.40,9.16)$ and right $(x, y=18.94,9.30)$ of the slit. The turn-number and $y_{\max }$ of the beam reaching $13 \mathrm{MeV}$ from those different initial positions in the slit of the ion source, and different initial phases $\left(-10^{\circ}, 0^{\circ}, 10^{\circ}\right)$, are tabulated in Table 1. Differences of $y_{\max }$ will provide estimates on the horizontal width of the beam near the stripper.

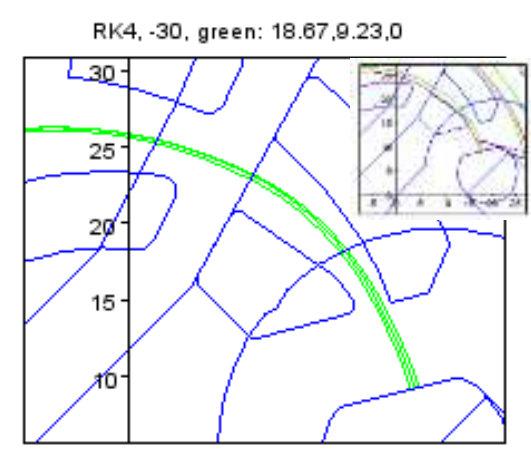

Fig. 5. The beam trajectory with an initial beam phase of $-30^{\circ}$, after the initial beam position was shifted $1 \mathrm{~mm}$ to the right. The inset figure shows the beam grazes the lower puller before the shift.

Table 1. Table of turn numbers $/ y_{\max }(\mathrm{mm})$ at $13 \mathrm{MeV}$, or maximum energies reached $<13 \mathrm{MeV}$, at various initial phases and initial positions in the ion source slit. Points (a) and (c) is $0.275 \mathrm{~mm}$ to the left and right, respectively, from the vertical axis of ion source slit. Point (b) is on the vertical axis.

\begin{tabular}{ccccc}
\hline \multirow{2}{*}{ Initial phase } & \multicolumn{4}{c}{ Turn-number/ $y_{\max }(\mathrm{mm})$ at $13 \mathrm{MeV}$} \\
& & $z=0$ & $z=2$ & $z=-2$ \\
\hline & a & $99 / 430$ & $12.9 \mathrm{MeV}$ & $12.9 \mathrm{MeV}$ \\
$-10^{\circ}$ & b & $96 / 426$ & $100 / 432$ & $99 / 432$ \\
& c & $95 / 421$ & $100 / 424$ & $100 / 432$ \\
& a & $97 / 428$ & $100 / 433$ & $100 / 442 *$ \\
$0^{\circ}$ & b & $96 / 426$ & $100 / 426$ & $12.8 \mathrm{MeV}$ \\
& c & $93 / 421$ & $96 / 426$ & $12.9 \mathrm{MeV}$ \\
& a & $96 / 427$ & $12.9 \mathrm{MeV}$ & $12.9 \mathrm{MeV}$ \\
$10^{\circ}$ & b & $96 / 423$ & $12.9 \mathrm{MeV}$ & $12.8 \mathrm{MeV}$ \\
& c & $96 / 423$ & $100 / 427$ & $12.9 \mathrm{MeV}$ \\
\hline
\end{tabular}

Table 1 shows that at initial beam phases of between $-10^{\circ}$ and $10^{\circ}$, the difference between the lowest and highest $y_{\max }$ is $12 \mathrm{~mm}$ (excluding one point with * mark); hence, it is the approximate horizontal width of the beam at $13 \mathrm{MeV}$. The lowest turn-number which reaches $13 \mathrm{MeV}$ is 92, while the highest is 100. From Fig. 3, it is found that the vertical width at these turn numbers is about $10 \mathrm{~mm}$, suggesting that the approximate beam width at $13 \mathrm{MeV}$ is about $10 \mathrm{~mm}$, in accordance with the size of the stripper designed for DECY-13.

Table 1 also shows that the beam with initial phase $0^{\circ}$ which came from from points (b) and (c) did not reach the intended energy of $13 \mathrm{MeV}$. The beam pulled out from the slit of the ion source exhibits an initial phase span of $180^{\circ}$. Allowing initial beam phases between $-10^{\circ}$ and $10^{\circ}\left(20^{\circ}\right.$ phase width) with some parts of the beam do not reach $13 \mathrm{MeV}$ or out of stripper size, it can be suggested that only about $10 \%$ of the beam coming out from the ion source will arrive at the stripper at $13 \mathrm{MeV}$. The $20^{\circ}$ phase width is better than what was reached in previous simulations, and similar to those found in KIRAMS. The $6 \mathrm{~mm}$ maximum distance between the ion source and puller also agrees with those found at several PET cyclotrons: KIRAMS $(3 \mathrm{~mm}, 45 \mathrm{kV}$ dee voltage), SKKUCY-9 $(3.5 \mathrm{~mm}, 40 \mathrm{kV}$ dee voltage [1]), Siemens Eclipse (3 mm, $39 \mathrm{kV}$ dee voltage), and GE (General Electric) Minitrace (1.5 mm, $30 \mathrm{keV}$ dee voltage).

\section{CONCLUSION}

At an accelerating RF frequency of 77.66 $\mathrm{MHz}$ and a peak dee voltage of $40 \mathrm{kV}$, the maximum distance between the ion source and the puller is $6 \mathrm{~mm}$. This agrees with those found at several PET cyclotrons. The advantage of closer distance, such as a higher beam percentage reaching the intended final energy, can be further simulated; however, it will also increase the chance of spark incidence (voltage breakdown), which may reduce the lifetime of an ion source.

The beam initial phase acceptance of $20^{\circ}$ is better than the result of previous simulations $\left(16^{\circ}\right)$. It yields an estimate that $10 \%$ of the ion source output will reach the stripper at the intended energy of $13 \mathrm{MeV}$. The beam width at this energy was estimated to be about $10 \mathrm{~mm}$ which agrees with the size of the beam stripper. 
The mechanism to obtain the position of the ion source to achieve the best final beam quality has also been simulated [10].

\section{ACKNOWLEDGMENT}

The authors thank Mr. Hari Suryanto from PTRR-BATAN and Mr. Hyun Wook Kim from Sungkyunkwan University, Korea, for providing information on the puller-to-ion-source distances.

\section{REFERENCES}

1. Silakhuddin and S. Santosa, Atom Indonesia 38 (2012) 7.

2. Taufik, A. Hermanto, P. Anggraita et al., Atom Indonesia 40 (2014) 69.

3. S.Y. Jung, H.W. Kim, M. Ghergherehchi et al.,
JINST (Journal of Instrumentation) 9 (2014) T04005.

4. D.H. An, I.S. Jung, J. Kang et al., Rev. Sci. Instrum. 79 (2008) 02A520.

5. B. Qin, K.F. Liu, Y.Z. Feng et al., Chinese Physics C 33 (2009) 682.

6. T. Zhang, M. Li, J. Zhong et al., Nuclear Instruments and Methods in Physics Research B 269 (2011) 2955.

7. T. Zhang, M. Li, J. Zhong et al., Nuclear Instruments and Methods in Physics Research A 676 (2012) 90.

8. E.V. Samsonov, B.N. Gikal, O.N. Borisov et al., Physics of Particles and Nuclei Letters 11 (2014) 158.

9. R.H. Landau, M.J. Paez and C.C. Bordeianu, A Survey Computational Physics, Princeton University Press, Princeton (2010) 178.

10. R.S. Darmawan, S. Santosa and Silakhuddin, Atom Indonesia 37 (2011) 113. 